\title{
An evaluation of the robustness of organ-at-risk recommendations made by GEC/ESTRO according to interobserver variability: a single-center experience
}

\author{
Rodolfo Chicas-Sett, MD 1.2,3, Francisco Celada-Alvarez, MD², Susana Roldán, MD², Asunción Torregrosa, MD4, \\ Jesus Betancourt, MD5 , Juan Bautista-Ballesteros, PhD², Dolores Farga, MD², Blanca lbañez, PhD², Alejandro Tormo, MD², \\ Jose Perez-Calatayud, PhD2,6 \\ 'Escuela de Doctorado, Universidad Católica de Valencia "San Vicente Mártir", Valencia, ${ }^{2}$ Department of Radiation Oncology, \\ La Fe Polytechnic and University Hospital, ${ }^{3}$ Department of Radiation Oncology, Recoletas Oncology Institute, Campo Grande Hospital, \\ Valladolid, "Department of Radiology, La Fe Polytechnic and University Hospital, ${ }^{4}$ Department of Urology, La Fe Polytechnic and University \\ Hospital, 'Department of Radiation Oncology, Clinica Benidorm, Benidorm, Alicante, Spain
}

\begin{abstract}
Purpose: Groupe Européen de Curiethérapie (GEC) and European Society for Radiotherapy \& Oncology (ESTRO) has proposed a rectal dose constraint of the most exposed 2-cc volume $\left(\mathrm{D}_{2 \mathrm{cc}}\right.$ of $\left.\leq 75 \mathrm{~Gy} \mathrm{EQD}_{2 \alpha / \beta}=3\right)$ during externalbeam plus high-dose-rate brachytherapy (HDR-BT) in localized prostate cancer patients. This study aimed to evaluate $D_{2 c c}$ for rectal contouring via interobserver variability.

Material and methods: Four blinded observers contoured rectums of 5 patients. Rectal contouring anatomical limits were determined through previous consensus. Dose-volume histogram $(\mathrm{DVH})$ dosimetric parameters $\left(\mathrm{D}_{0.1 \mathrm{cc}}, \mathrm{D}_{1 \mathrm{cc} \mathrm{c}^{\prime}}\right.$ and $\left.\mathrm{D}_{2 c c}\right)$ were analyzed according to GEC/ESTRO recommendations and subjected to intra- and interobserver comparisons. Latter comparisons involved coefficients of variation. For each parameter, the mean, standard deviation (SD), and range were evaluated. The effect of interobserver variation on total dose was analyzed by estimating the biologically equivalent rectal dose $\left(\mathrm{EQD}_{2 \alpha / \beta}=3\right)$.

Results: Interobserver coefficients of variation for $\mathrm{D}_{0.1 \mathrm{c} c^{\prime}} \mathrm{D}_{1 \mathrm{cc}}$ and $\mathrm{D}_{2 \mathrm{cc}}$ were $5.7 \%, 4.5 \%$, and $4 \%$, respectively. The highest interobserver rectal delineation variation yielded a rectal dose difference up to $5.8 \mathrm{~Gy} \mathrm{EQD}_{2}$. Estimated intraobserver variation for the reported $\mathrm{D}_{2 c c}$ was $5.5 \%$ in the worst-case scenario (non-significant).

Conclusions: We observed acceptable interobserver variability in $\mathrm{EQD}_{2}$ for $\mathrm{D}_{2 \mathrm{cc}}$, with strong impacts on clinical threshold levels $\left(\mathrm{D}_{2 \mathrm{cc}} \leq 75 \mathrm{~Gy} \mathrm{EQD}_{2}\right)$ in some cases. This small, single-center analysis will be extended in a multicenter study.

Key words: brachytherapy, GEC-ESTRO, high-dose-rate, prostate cancer, organs at risk.

\section{Purpose}

High-dose-rate brachytherapy (HDR-BT), defined by Morton et al. as a method of conformal dose escalation to the prostate [1], involves the placement of sealed sources of radiation in contact with a tumor using after-loading devices. This type of therapy plays an important role in the management of prostate cancer. Notably, dose escalation strategies, which allow the delivery of high radiation doses, have yielded improved local control in patients with prostate cancer. Accordingly, HDR-BT is considered a very acceptable option when used in combination with external beam radiotherapy (EBRT) [2]. A significant number of EBRT and HDR-BT boost studies have reported biochemical relapse-free survival (BRFS) rates of $63-97 \%$ in interme-

diate and high-risk patients [3]. Hoskin et al., in a randomized phase III trial, observed a significant improvement in BRFS with EBRT + HDR-BT versus EBRT alone, along with a $31 \%$ reduction in the risk of recurrence [4].

In addition, HDR-BT monotherapy is gaining relevance as a promising treatment for prostate cancer. However, its administration is under protocol and the majority of related studies have involved a relatively short follow-up period [5]. In 2013, Zamboglou et al. published a study with the longest follow-up period to date (52.8 months), and reported biochemical control rates exceeding $90 \%$ (including intermediate and high-risk groups) [6]. In 2015, Kukiełka et al. published local control outcomes as high as $96.9 \%$ [7]. Similar urinary toxicity has been observed in patients treated with HDR monotherapy [8].
\end{abstract}

Address for correspondence: Rodolfo Chicas-Sett, MD, Department of Radiation Oncology, La Fe Polytechnic and University Hospital, Avenida Fernando Abril Martorell 106, Torre B, Planta Baja, 46026, Valencia, Spain, phone: +34 961245098, e-mail: rchicas@ufm.edu
Received: 28.04 .2016

Accepted: 20.07 .2016

Published: 24.08.2016 
The primary goal of HDR-BT is the delivery of a high radiation dose to the target tissue; however, this goal is restricted by the presence of the surrounding organs at risk (OAR) such as the rectum, which limit the planned total dose for a definitive treatment [9]. A high dose to the rectum may cause adverse effects such as local inflammation, fibrosis, telangiectasia, ulceration, necrosis, and fistula, which are directly related to the magnitude of the administered dose [10]. Although rare, rectal complications after combined EBRT and HDR-BT have been reported and cannot be completely prevented. The majority of studies have reported grade 2 toxicity with this combination therapy. Proctitis, rectal ulceration, and fistula formation have also been described [11].

The effects of the doses to the target and normal tissues can be analyzed and calculated by planning systems from dose-volume histograms (DVH). Dose-volume histogram values can be expressed in absolute (cc) or relative volumes (\%). The usage of different doses, techniques, and fractionation schedules among departments, however, may present a challenge in the identification of universal quality parameters for the evaluation of brachytherapy treatment plans [12]. In this light, various parameters and indices for OAR documentation (most exposed 0.1-, $1-, 2-, 5-, 10-\mathrm{cc}$ volumes; $\mathrm{D}_{0.1 \mathrm{cc},} \mathrm{D}_{1 \mathrm{cc}}, \mathrm{D}_{2 \mathrm{cc}}, \mathrm{D}_{5 \mathrm{cc}}, \mathrm{D}_{10 \mathrm{cc}}$ respectively) and the target volume $\left(\mathrm{V}_{100}, \mathrm{~V}_{150}\right.$, and $\mathrm{V}_{200}$, or percentages of the clinical target volume [CTV] receiving $100 \%, 150 \%$, and $200 \%$ of the prescribed dose, respectively; $D_{100}$ and $D_{90}$, or the doses covering $100 \%$ and $90 \%$ of the CTV, respectively) have been proposed in the context of Groupe Européen de Curiethérapie (GEC) and European Society for Radiotherapy \& Oncology (ESTRO) recommendations for the treatment of cervical cancer [10]. These parameters were subsequently extrapolated, used, and suggested as comparable universal dosimetric parameters in the recommendations by Hoskin et al. regarding HDR-BT for prostate cancer $[13,14]$.

Because the use of different EBRT and HDR-BT scheme results in considerable dose heterogeneity, it is difficult to obtain a generalized OAR constraint. The GEC/ESTRO accordingly recommends the use of an absolute dose-volume constraint expressed in $\mathrm{Gy}_{\alpha / \beta=3}$ for every fractionation based on an $\mathrm{EQD}_{2}$ total dose [13]. The $\mathrm{D}_{2 \mathrm{cc}} \leq 75 \mathrm{~Gy} \mathrm{EQD}_{2}$ has been indicated for specific cases involving the rectum, and has also been supported by Crook et al., who reported absolute volumes rather than relative doses because the latter are subjective and very sensitive to the number of contoured slides and contoured shape of the wall [15].
Interobserver variation, when contouring clinical target volumes (CTVs), is known as an important source of systematic error in the radiotherapy treatment process. Therefore, several studies have assessed interobserver variability. For example, in gynecological brachytherapy, delimitation of the high-risk CTV has been used to demonstrate acceptable interobserver variability $[16,17,18,19]$. However, limited data are available on the impacts of contouring errors on doses to the OARs. Given the above issues, this pilot study aimed to determine the degree of interobserver variability with regard to rectal contouring during HDR-BT treatment planning, and to analyze the robustness of $D_{2 c c}$ as an acceptable parameter according to the GEC/ESTRO recommendations in our Radiation Oncology Department.

\section{Material and methods}

This single-center retrospective study included 5 sets of ultrasound (US) images from prostate cancer patients that were used for HDR-BT planning. Four expert physicians performed rectal contouring.

\section{Study cases}

The HDR-BT treatment planning data of 5 patients treated with combined radiotherapy (HDR-BT and EBRT) at La Fe Polytechnic and University Hospital were included. All patients were diagnosed with prostate adenocarcinoma and treated according to the same treatment plan. These patients were selected to provide a range of different prostate sizes for this study (Table 1), as well as for other characteristics.

\section{Treatment planning}

HDR-BT treatment planning was performed on an Oncentra Prostate ${ }^{\circledR}$ planning device (version 4.2; Nucletron, an Elekta company, Veenendaal, Netherlands). External beam radiotherapy planning was performed on an Eclipse planning device (version 13.0; Varian Medical Systems, Palo Alto, CA, USA).

The treatment was designed such that HDR-BT was performed first, followed by computed tomography (CT) simulation 2 weeks later and EBRT after an additional 2-week interval (i.e., 4 weeks after HDR-BT). In the instance of a complication that would prohibit HDR-BT, this plan would allow a continuous high dose treatment during EBRT.

Table 1. Baseline characteristics of the patient group

\begin{tabular}{lccccc} 
Case/Patient & Prostate volume $\left(\mathrm{mm}^{3}\right)$ & Age & PSA $(\mathrm{ng} / \mathrm{ml})$ & Tumor & Gleason score \\
\hline 1 & 35.71 & 63 & 5.17 & T3a & 6 \\
\hline 2 & 28.14 & 72 & 20.40 & T3a & 7 \\
\hline 3 & 44.78 & 78 & 27.37 & T1 & 7 \\
\hline 4 & 39.47 & 71 & 30.00 & T2 & 6 \\
\hline 5 & 53.70 & 70 & 9.20 & T2 & 7
\end{tabular}

PSA - prostate-specific antigen 
Brachytherapy was administered in a 15 Gy single fraction, and the intraoperative procedure was based on US imaging findings. The patient was placed in a lithotomy position, and transversal images were captured in $1 \mathrm{~mm}$ slices using a trans-rectal ultrasound (TRUS) probe. The CTV was defined as the entire prostate gland, and the planning target volume (PTV) was defined as the CTV plus a $3 \mathrm{~mm}$ margin (except in the rectal and vesical directions). The urethra and rectum were contoured as OARs.

Dose distributions were optimized by determining the dwell positions and dwell times for the source within each needle and calculating the $\mathrm{D}_{90}$ for the target volume and $\mathrm{D}_{2 \mathrm{cc}}$ for the OARs. The needles were inserted through a transperineal template, using live TRUS images for guidance. Treatment was delivered using a HDR ${ }^{192}$ Ir source. Needles were removed after treatment, and 4 gold fiducials were implanted for EBRT image guidance.

External beam radiotherapy was planned using CT images. The CTV was defined as the prostate gland, and the PTV was defined as the CTV with a $5 \mathrm{~mm}$ margin in all directions except posteriorly, where the margin was $4 \mathrm{~mm}$. Volumetric modulated arc therapy (RapidArc, Varian Medical Systems) and image-guided radiation therapy were used, and daily cone beam CT or orthogonal $\mathrm{kV}$ images were combined. The prescribed dose was defined such that $95 \%$ of the PTV should receive at least $95 \%$ of the prescribed dose (46 Gy).

\section{Contouring}

An expert group comprising 2 radiation oncologists, 1 radiologist, and 1 urologist usually involved in prostate brachytherapy and prostate US was established. This group had previously determined rectal delineation criteria in consensus.

Two identical US image sets were generated from the original HDR-BT contouring plan. Image assembly was anonymized to avoid bias. Ultrasound image sets were obtained with a Primus $6.5 \mathrm{MHz}$ ultrasound device (Hitachi, Ltd., Tokyo, Japan). Axial images of the prostate were captured from the base through the apex. Rectums were contoured on 5 image sets by 4 blinded observers.

Each observer contoured the rectal wall on the axial slides in $5 \mathrm{~mm}$ slide increments according to the previously established consensus criteria. All observers were blinded to the other physicians' contours and were only provided the urethra contour as a reference for longitudinal rectum delineation.

In our study, the radiologic anatomic boundaries of the rectum, according to the previous consensus, were: 1) $10 \mathrm{~mm}$ upward of the CTV volume in the cranial direction; 2) $10 \mathrm{~mm}$ below of the CTV volume in the caudal direction; 3) the posterior layer of Denonvillier's fascia in the anterior direction; and 4) the rectal wall visible on the US screen in the posterior direction (see Figures 1 and 2).

\section{Study design}

Four observers delineated the rectum on 5 US image sets from 5 prostate cancer patients. The observers repeated the delineation procedure twice at a 1-week interval.
Forty rectal contours $(4$ observers $\times 5$ patients $\times 2$ records for each case) were created and made available for analysis. Only the main investigator, who supervised the delineations performed by the 4 observers but did not actively participate in the delineation process, controlled the data registry and adequate identification of the patients and data. Figure 3 presents the scheme of the study.

\section{Dose volume histogram analyses}

Dose-volume histograms were used to evaluate plans according to the GEC/ESTRO recommendations on HDR-BT for prostate cancer [13]. For each patient, the plan from a single HDR-BT fraction selected for contouring was used to calculate the DVH parameters. Using the source configuration from the optimized plans, the $\mathrm{D}_{0.1 \mathrm{cc}}$ $D_{1 \mathrm{cc}}$ and $\mathrm{D}_{2 \mathrm{cc}}$ for the rectum were calculated for the observed contour set for each case.

For each DVH parameter, the mean value and standard deviation (SD) were calculated for each observer. To

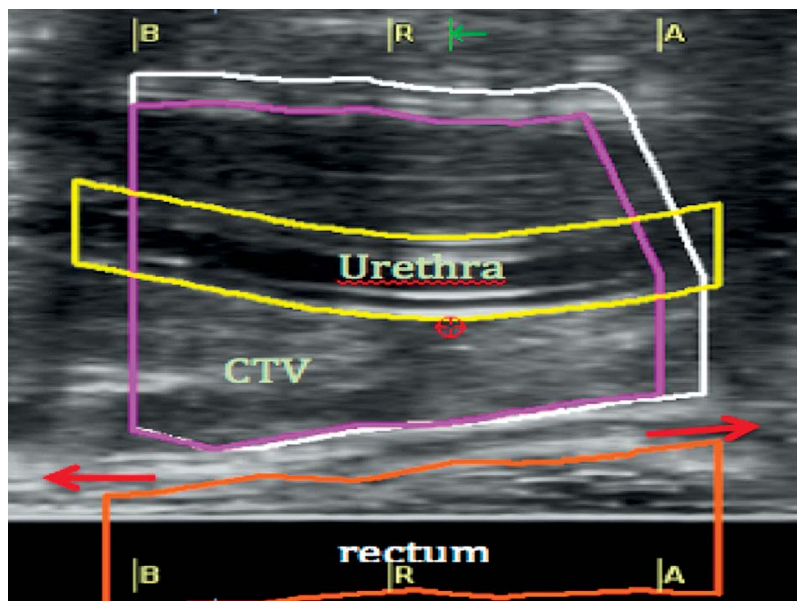

Fig. 1. Sagittal ultrasound (US) image showing the prostate and different rectal contours. The rectal structure includes a $10 \mathrm{~mm}$ margin that is craniocaudal with respect to the clinical target volume (CTV; red arrow). The shaded region (orange) indicates the rectal contour delineated by each observer

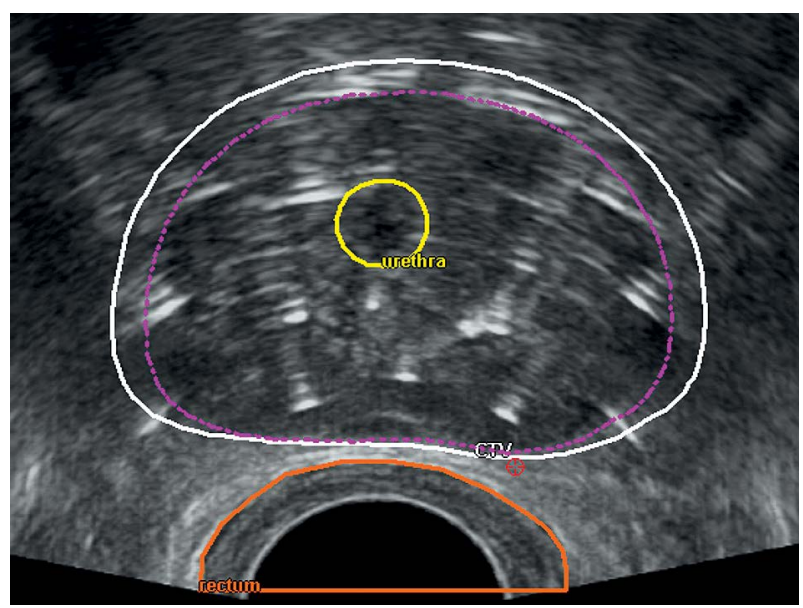

Fig. 2. Transverse ultrasound image showing an example of a rectal contour (orange line) 


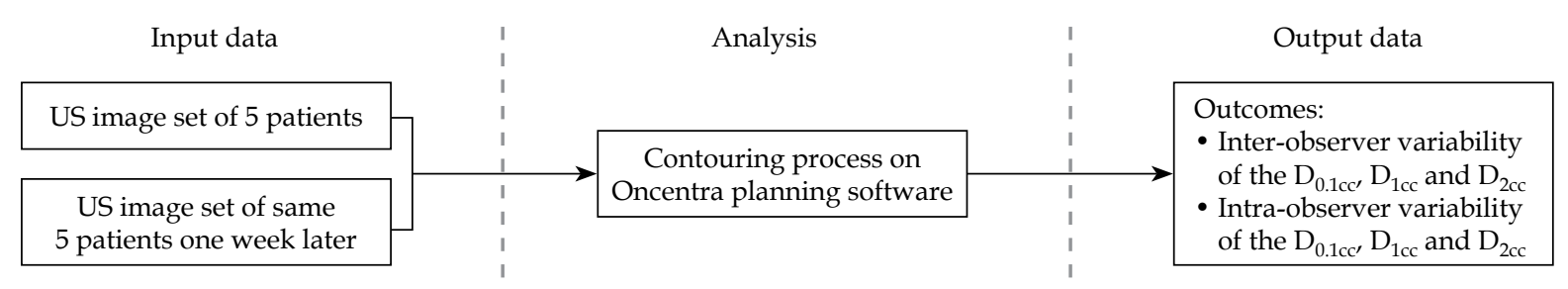

Fig. 3. Scheme of the study design. US - ultrasound; $\mathrm{D}_{0.1 \mathrm{c} c^{\prime}} \mathrm{D}_{1 \mathrm{cc}}$ and $\mathrm{D}_{2 \mathrm{c} \mathrm{c}^{\prime}}$ most exposed 0.1-, 1-, and 2-cc volumes of the rectum

compare variability in the different DVH parameters according to Duane et al., the coefficient of variation (COV) was used to provide a measure of the data dispersion as a proportion of the mean [19].

The 4 observers determined the means and SDs of the 2 measurements recorded for the matched US image sets for each parameter. The overall mean of these 4 measurements was then calculated for each patient. The interobserver COV was obtained by calculating the ratio of the SD to the mean for each patient. In the end, the overall COV for the 5 patients was calculated to provide a measure of interobserver variation across the entire group [19]. For the 5 patients, the differences in dose values between duplicated US image sets were analyzed using the non-parametric Friedman test. Statistical analyses were conducted using XLSTAT software (version 2014.6.01; Addinsoft, Paris, France).

The impact of contouring uncertainties on the total dose delivered to the rectum was evaluated by estimating the total dose (EBRT + HDR-BT), assuming that the rectum received the prescribed EBRT dose (46 Gy) as described above. All dose values were biologically normalized to an $\mathrm{EQD}_{2}$ expressed in units of $\mathrm{Gy}_{\alpha / \beta=3}$.

The intraobserver COV was calculated to determine intraobserver variability. This value was defined as 2 SDs of the value resulting from the following equation: absolute value [first measurement - second measurement]/ mean measurement, where the absolute value is the absolute difference between the 2 measurements made by the same observer [19]. For the 5 patients, the differences in dose values between duplicated US image sets were analyzed using the Wilcoxon signed-rank test. Statistical analyses were conducted using XLSTAT software (version 2014.6.01; Addinsoft, Paris, France). The test revealed no statistically significant differences in the $\mathrm{D}_{0.1 \mathrm{cc}}, \mathrm{D}_{1 \mathrm{cc}}$ and $\mathrm{D}_{2 \mathrm{cc}}$ dose parameters $(p=0.059,0.418$, and 0.281 , respectively).

\section{Results}

\section{Interobserver variation: impact on reported dose volume histogram}

The mean reported $\mathrm{D}_{0.1 \mathrm{cc}} \mathrm{D}_{1 \mathrm{cc} \mathrm{c}^{\prime}}$ and $\mathrm{D}_{2 \mathrm{cc}}$ values for the rectum from 2 sessions of contouring are summarized for each patient in Table 2.

The overall mean of the interobserver COV for all patients and all observers is presented in Table 3. Greater interobserver variation was observed for $\mathrm{D}_{0.1 \mathrm{cc}}$. However, the larger SD of 2.62 for case 5, relative to the SDs of 0.280.5 for the other cases, might be explained by interobserver variation. The global test revealed significant differenc- es in the $D_{0.1 c c}, D_{1 c c}$ and $D_{2 c c}$ for the rectum among the observers $(p<0.05)$ using the Friedman test.

\section{Interobserver variation: impact on evaluated total rectum dose}

The greatest interobserver variation in the $\mathrm{D}_{0.1 \mathrm{cc}}$ group was $16.8 \%$ greatest for case 5 , with $D_{0.1 c c}$ values ranging from 1.47-18.42 Gy, indicating that the potential total reported rectum $\mathrm{D}_{0.1 \mathrm{cc}}$ ranged from 82.55-98.22 Gy. Similar magnitudes of interobserver variation were observed for $D_{1 \mathrm{cc}}$ and $D_{2 \mathrm{cc}}$. The greatest interobserver variations for $\mathrm{D}_{1 \mathrm{cc}}$ and $\mathrm{D}_{2 \mathrm{cc}}$ were also observed in case 5 , with values of $6.4 \%$ and $4.5 \%$, respectively. The reported range of variability in $\mathrm{D}_{2 \mathrm{cc}}$ was $0.61-2.53 \mathrm{~Gy}$, indicating that the potential total reported rectal $\mathrm{D}_{2 \mathrm{cc}}$ ranged from 71.10-77.25 Gy. The higher interobserver variability described above for $\mathrm{D}_{2 \mathrm{cc}}$ corresponds to a worst-case scenario of a rectal contouring variation that might result in a recorded dose difference of up to $5.8 \mathrm{~Gy}$, as shown in Table 4.

\section{Intraobserver variation: impact on reported dose volume histogram parameters}

The intraobserver variation for the reported $D_{2 c c}$ ranged from $2.5 \%$ to $6.3 \%$. Variations in rectal delineation were consistent for each patient. Given that the $\mathrm{D}_{0.1 \mathrm{cc}}$ $\mathrm{D}_{1 \mathrm{cc}}$, and $\mathrm{D}_{2 \mathrm{cc}}$ values for observer 1 were similar in both US image sets, we tested for differences in dose values between duplicate US image sets for this observer using the Wilcoxon signed-rank test.

\section{Discussion}

To date, advances in technology and clinical experience have led to major progress in HDR-BT for prostate cancer. However, the delineation of target volumes and OARs remains dependent on the observer. Variability in the delineation of these elements can limit the brachytherapy dose distribution, representing a main source of uncertainty that can impact clinical and treatment outcomes $[20,21,22,23]$. Hence, quantification of the dosimetric impact of this delineation variability is necessary.

Studies of variability in contouring of target volumes and OARs are well represented in the literature [24]. Many such studies (e.g., a study by Wong et al. [25]) indicate that delineation guidelines could improve interobserver homogeneity. Furthermore, in other studies (such as that Buch et al. [26]) the use of high-resolution image as contrast enhanced magnetic resonance imaging could improve the dosimetry to OARs. Although the GEC/ ESTRO recommendations for HDR-BT of prostate cancer 
Table 2. Values of the most exposed $0.1,1$, and 2-cc volumes $\left(D_{0.1 c c}, D_{1 c c}\right.$, and $D_{2 c c}$, respectively) of the rectum for each patient and observer based on a single 15 Gy high dose rate brachytherapy plan. Data are shown as the means of values obtained at 2 different time points. Interobserver mean, range, and standard deviation for each patient are represented in the right side $D_{0.1 c c}(G y)$

\begin{tabular}{|c|c|c|c|c|c|c|c|}
\hline Patient & Observer A & Observer B & Observer C & Observer D & Mean & Range & SD \\
\hline \multicolumn{8}{|c|}{$D_{0.1 c c}(G y)$} \\
\hline 1 & 12.41 & 11.98 & 12.30 & 11.79 & 12.12 & $11.79-12.41$ & 0.28 \\
\hline 2 & 12.48 & 12.16 & 12.63 & 11.79 & 12.27 & $11.79-12.63$ & 0.37 \\
\hline 3 & 12.45 & 12.45 & 12.68 & 11.96 & 12.39 & $11.96-12.68$ & 0.30 \\
\hline 4 & 13.62 & 12.43 & 13.22 & 12.91 & 13.04 & $12.43-13.62$ & 0.50 \\
\hline 5 & 13.56 & 15.65 & 19.16 & 13.65 & 15.51 & $13.56-19.16$ & 2.62 \\
\hline \multicolumn{8}{|l|}{$D_{1 c c}(G y)$} \\
\hline 1 & 10.35 & 11.47 & 10.50 & 10.13 & 10.61 & $10.13-11.47$ & 0.59 \\
\hline 2 & 10.43 & 11.13 & 10.54 & 9.84 & 10.49 & $9.84-11.13$ & 0.53 \\
\hline 3 & 10.89 & 11.52 & 10.82 & 10.50 & 10.93 & $10.50-11.52$ & 0.43 \\
\hline 4 & 11.40 & 11.60 & 11.51 & 11.23 & 11.44 & $11.23-11.60$ & 0.16 \\
\hline 5 & 11.61 & 13.15 & 12.98 & 11.79 & 12.38 & $11.61-13.15$ & 0.79 \\
\hline \multicolumn{8}{|l|}{$D_{2 c c}(G y)$} \\
\hline 1 & 9.69 & 9.37 & 9.58 & 9.24 & 9.47 & $9.24-9.69$ & 0.21 \\
\hline 2 & 9.36 & 9.12 & 9.48 & 8.72 & 9.17 & $8.72-9.48$ & 0.34 \\
\hline 3 & 9.82 & 9.92 & 9.86 & 9.61 & 9.80 & $9.61-9.92$ & 0.13 \\
\hline 4 & 10.32 & 9.71 & 10.49 & 10.22 & 10.19 & $9.71-10.49$ & 0.34 \\
\hline 5 & 10.62 & 11.13 & 11.76 & 10.81 & 11.08 & $10.62-11.76$ & 0.50 \\
\hline
\end{tabular}

Table 3. Overall interobserver coefficients of variation (\%) for the recorded most exposed $0.1,1$, and 2 cc volumes $\left(D_{0.1 c c}, D_{1 c c}\right.$, and $D_{2 c c}$, respectively) of the rectum based on the single 15 Gy high-doserate brachytherapy plan

\begin{tabular}{lccc} 
& \multicolumn{3}{c}{ Dosimetric parameters } \\
\cline { 2 - 4 } & $\mathrm{D}_{0.1 \mathrm{cc}}$ & $\mathrm{D}_{1 \mathrm{cc}}$ & $\mathrm{D}_{2 \mathrm{cc}}$ \\
\hline Coefficient of variation (COV) & 5.71 & 4.46 & 4.06
\end{tabular}

have been published and updated in 2013 with the inclusion of $D_{2 c c}$ and $D_{0.1 c c}$ doses for the rectum [13], to our knowledge, we are the first group to report the effects of interobserver and intraobserver variability on rectal delineation in the context of HDR-BT treatment for prostate cancer.

In the present study, despite the use of contouring consensus-based rectal delineation criteria, significant interobserver differences were detected in the dose parameters; specifically, the average interobserver COVs for $\mathrm{D}_{0.1 \mathrm{cc}}, \mathrm{D}_{1 \mathrm{cc},}$ and $\mathrm{D}_{2 \mathrm{cc}}$ were $5.71 \%, 4.46 \%$, and $4.06 \%$, respectively. Although rectal contouring was consistent among the observers, caudal limit contouring was difficult because of the varied interpretations of the rectal border and delimitation of the sphincter muscle. This difficulty was
Table 4. Range (standard deviation) of the biologically equivalent dose $\left(\mathrm{EQD}_{2}\right)$ of the most exposed 2 cc volume $\left(D_{2 c c}\right)$ of the rectum for each patient, based on the single 15 Gy high-dose-rate brachytherapy plan plus $46 \mathrm{~Gy}$ external beam radiotherapy

\begin{tabular}{lcc} 
Case/Patient & $D_{2 c c}$ (Gy) & Dose difference (Gy) \\
\hline 1 & $68.60-70.62(0.91)$ & 2.0 \\
\hline 2 & $66.45-69.67(1.41)$ & 3.2 \\
\hline 3 & $70.25-71.66(0.61)$ & 1.4 \\
\hline 4 & $70.70-74.30(1.54)$ & 3.6 \\
\hline 5 & $74.95-80.71(2.53)$ & 5.8
\end{tabular}

clearly observed in the analysis of case 5 , wherein a COV of $16.8 \%$ was calculated for $\mathrm{D}_{0.1 \mathrm{cc}}$. This variability is reasonable because $D_{0.1 c c}$ represents the smallest dose point of the largest dose near the rectum wall and is therefore highly sensitive to inaccuracies in contouring. No statically significant intraobserver differences in the dose parameters were reported.

Evidence for variations in the delineation of OARs has been primarily reported from gynecological studies using the GEC/ESTRO recommendations. Hellebust et al. reported interobserver delineation variability of $5-8 \%$ for 
the $\mathrm{D}_{2 \mathrm{cc}}$ of the rectum in a study of the dosimetric impact of magnetic resonance imaging-based cervical cancer brachytherapy [27]. Saarnak et al. [28] reported a higher variability rate (approximately $11 \%$ ). In our study, we obtained an interobserver $\mathrm{COV}<5 \%$ for $\mathrm{D}_{2 \mathrm{cc}}$ although random dosimetric variations were observed in individual cases. The low dose variability observed in our study might be associated with proper training of the physicians and implementation of the consensus contouring guidelines. However, no previously published data regarding HDRBT for prostate cancer were available for comparison.

The impact on the total received dose (HDR-BT + EBRT) corresponded with an $\mathrm{EQD}_{2}$ range of 1.4-5.8 Gy. This difference in doses was similar to the range published by Hellebust $\left(2-3 \mathrm{~Gy}_{\alpha / \beta=3}\right)$ [27]. Nesvacil et al. [29] reported a slightly higher inter-fractional dose difference range of 4-8 Gy EQD 2 for OARs in a multicenter study.

Regarding rectum delineation, the observers emphasized the quality of the US images but also noted difficulty with correctly contouring the final area of the rectum proximal to the anus in some cases. In one particular case, the large prostate volume led to uncertainty when contouring the anterior limit of the rectum proximal to the prostate, although this difficulty might have been limited to this particular case or to inherent uncertainties of the observers. This incident was relevant to the dosimetric analysis because the upper limit of the $\mathrm{EQD}_{2}(5.8 \mathrm{~Gy})$ represents the total dose received by the rectum at a dose range of 74.95-80.71 Gy, which exceeds the recommended dose according to the GEC/ESTRO.

The impact of dosimetric variability is more significant in high dose regions near the target volume than in low dose regions. However, whereas the OARs are associated with low doses, factors such as interobserver variability in delineation could lead to severe toxicity of the OARs. George et al. [30] referred to side effects after radiotherapy (EBRT and brachytherapy) for cervical carcinoma; specifically, the presence of telangiectasias correlated with the $2-\mathrm{cm}^{3}$ high dose rectal volume, and ulcerations were limited to the small $0.1-\mathrm{cm}^{3}$ high dose volume. In our study, we observed dose uncertainties up to $5.8 \mathrm{~Gy}$, which was higher than the range of 2-3 Gy published by Georg et al. [30] (no correspondence with critical consequences). Nevertheless, dosimetric uncertainties become important with respect to interobserver variability when the OAR doses approach the maximum limit in an attempt to optimize the brachytherapy treatment.

The sample size is a limitation in this study. It is relatively small and a larger or multicenter study should be made before extrapolation to population. However, we believe that the results obtained will establish a starting point of the robustness of $\mathrm{D}_{2 \mathrm{cc}}$ as an acceptable parameter according to the GEC/ESTRO recommendations in our experienced Radiation Oncology Department.

\section{Conclusions}

In general, we obtained acceptable interobserver variability in the $\mathrm{EQD}_{2}$ for the reported $\mathrm{D}_{2 \mathrm{cc}}$ although a high impact on clinical threshold levels $\left(\mathrm{D}_{2 c \mathrm{c}} \leq 75 \mathrm{~Gy} \mathrm{EQD}_{2}\right)$ was present in some cases. Interobserver variability was lowest for $D_{2 c c}(<5 \%)$, in agreement with previously published studies on brachytherapy for gynecological cancers. In our study, the impact of interobserver variation on the $\mathrm{EQD}_{2}$ for the reported $D_{2 c c}$ had the potential to yield a worst-case scenario dose difference of up to $5.8 \mathrm{~Gy}_{\alpha / \beta=3}$. Although the GEC/ESTRO recommendations provide a common language for reporting dose information, future studies are needed to identify correlations of interobserver delineation variability with adverse effects and clinical outcomes.

The outcomes obtained in this pilot study should be validated. In addition, a multicenter study is needed as a follow-up to this small, single-center study.

\section{Disclosure}

Authors report no conflict of interest.

\section{References}

1. Morton GC. High-dose-rate brachytherapy boost for prostate cancer: rationale and technique. J Contemp Brachytherapy 2014; 6: 323-330.

2. Yamada Y, Rogers L, Demanes DJ et al. American Brachytherapy Society consensus guidelines for high-dose-rate prostate brachytherapy. Brachytherapy 2012; 11: 20-32.

3. De Bari B, Daidone A, Alongi F. Is high dose rate brachytherapy reliable and effective treatment for prostate cancer patients? A review of the literature. Crit Rev Oncol Hematol 2015; 94: 360-370.

4. Hoskin PJ, Rojas AM, Bownes PJ et al. Randomized trial of external beam radiotherapy alone or combined with highdose-rate brachytherapy boost for localized prostate cancer. Radiother Oncol 2012; 103: 217-222.

5. Demanes DJ, Martinez AA, Ghilezan M et al. High-dose-rate monotherapy: safe and effective brachytherapy for patients with localized prostate cancer. Int J Radiat Oncol Biol Phys 2011; 81: 1286-1292.

6. Zamboglou N, Tselis N, Baltas D et al. High-dose-rate interstitial brachytherapy as monotherapy for clinically localized prostate cancer: treatment evolution and mature results. Int J Radiat Oncol Biol Phys 2013; 85: 672-678.

7. Kukiełka AM, Dąbrowski T, Walasek T et al. High-dose-rate brachytherapy as monotherapy for prostate cancer - Single-institution results of the extreme fractionation regimen. Brachytherapy 2015; 14: 359-365.

8. Cendales R, Alwers E, Cifuentes J et al. High-dose-rate brachytherapy delivered in two fractions as monotherapy for low-risk prostate cancer. J Contemp Brachytherapy 2015; 7: 10-16.

9. Bölling T, Moustakis C, Elsayed H et al. Rectum dose reduction and individual treatment plan optimization for high-dose-rate prostate brachytherapy. Brachytherapy 2007; 6: 280-285.

10. Pötter R, Haie-Meder C, Van Limbergen E et al. Recommendations from gynaecological (GYN) GEC ESTRO working group (II): concepts and terms in 3D image-based treatment planning in cervix cancer brachytherapy-3D dose volume parameters and aspects of 3D image-based anatomy, radiation physics, radiobiology. Radiother Oncol 2006; 78: 67-77.

11. Ghilezan M, Kestin L, Gustafson G et al. A comprehensive toxicity analysis of 1232 prostate cancer patients treated with pelvic external beam radiotherapy (EBRT) with high dose rate (HDR) brachytherapy boost, adaptive EBRT (ART), or brachytherapy alone (BT). Int J Radiat Oncol Biol Phys 2006; 66: S59-S60.

12. Kirisits C, Goldner G, Berger D et al. Critical discussion of different dose-volume parameters for rectum and urethra in prostate brachytherapy. Brachytherapy 2009; 8: 353-360. 
13. Hoskin PJ, Colombo A, Henry A et al. GEC/ESTRO recommendations on high dose rate afterloading brachytherapy for localised prostate cancer: an update. Radiother Oncol 2013; 107: 325-332.

14. Kovács G, Pötter R, Loch T et al. GEC/ESTRO-EAU recommendations on temporary brachytherapy using stepping sources for localised prostate cancer. Radiother Oncol 2005; 74: 137-148.

15. Crook JM, Potters L, Stock RG et al. Critical organ dosimetry in permanent seed prostate brachytherapy: defining the organs at risk. Brachytherapy 2005; 4: 186-194.

16. Petric $P$, Dimopoulos J, Kirisits $C$ et al. Inter- and intraobserver variation in HR-CTV contouring: intercomparison of transverse and paratransverse image orientation in 3D-MRI assisted cervix cancer brachytherapy. Radiother Oncol 2008; 89: 164-171.

17. Dimopoulos JC, De Vos V, Berger D et al. Inter-observer comparison of target delineation for MRI-assisted cervical cancer brachytherapy: application of the GYN GEC-ESTRO recommendations. Radiother Oncol 2009; 91: 166-172.

18. Petrič P, Hudej R, Rogelj P et al. Uncertainties of target volume delineation in MRI guided adaptive brachytherapy of cervix cancer: a multi-institutional study. Radiother Oncol 2013; 107: 6-12.

19. Duane FK, Langan B, Gillham C et al. Impact of delineation uncertainties on dose to organs at risk in CT-guided intracavitary brachytherapy. Brachytherapy 2014; 13: 210-218.

20. Njeh CF. Tumor delineation: The weakest link in the search for accuracy in radiotherapy. J Med Phys 2008; 33: 136-140.

21. Fotina I, Lütgendorf-Caucig C, Stock M et al. Critical discussion of evaluation parameters for inter-observer variability in target definition for radiation therapy. Strahlenther Onkol 2012; 188: 160-167.

22. Allozi R, Li XA, White J et al. Tools for consensus analysis of experts' contours for radiotherapy structure definitions. Radiother Oncol 2010; 97: 572-578.

23. Weiss E, Richter S, Krauss $\mathrm{T}$ et al. Conformal radiotherapy planning of cervix carcinoma: differences in the delineation of the clinical target volume. A comparison between gynaecologic and radiation oncologists. Radiother Oncol 2003; 67: 87-95.

24. Collier DC, Burnett SS, Amin M et al. Assessment of consistency in contouring of normal-tissue anatomic structures. J Appl Clin Med Phys 2003; 4: 17-24.

25. Wong EK, Truong PT, Kader HA et al. Consistency in seroma contouring for partial breast radiotherapy: impact of guidelines. Int J Radiat Oncol Biol Phys 2006; 66: 372-376.

26. Buch K, Morancy T, Kaplan I et al. Improved dosimetry in prostate brachytherapy using high resolution contrast enhanced magnetic resonance imaging: a feasibility study. J Contemp Brachytherapy 2015; 6: 337-343.

27. Hellebust TP, Tanderup K, Lervåg $C$ et al. Dosimetric impact of interobserver variability in MRI-based delineation for cervical cancer brachytherapy. Radiother Oncol 2013; 107: 13-19.

28. Saarnak AE, Boersma M, van Bunningen BN et al. Inter-observer variation in delineation of bladder and rectum contours for brachytherapy of cervical cancer. Radiother Oncol 2000; 56: 37-42.

29. Nesvacil N, Tanderup K, Hellebust TP et al. A multicentre comparison of the dosimetric impact of inter- and intra-fractional anatomical variations in fractionated cervix cancer brachytherapy. Radiother Oncol 2013; 107: 20-25.

30. Georg P, Pötter R, Georg D et al. Dose effect relationship for late side effects of the rectum and urinary bladder in magnetic resonance image-guided adaptive cervix cancer brachytherapy. Int J Radiat Oncol Biol Phys 2012; 82: 653-657. 